\title{
Comunicación
}

\section{Obstrucción Gastrointestinal por Cuerpos Extraños en una Tortuga Charapa (Podocnemis expansa) Mantenida en Cautiverio}

\author{
Gastrointestinal Obstruction by Foreign Bodies in a Captive Giant South \\ American Turtle (Podocnemis expansa)
}

\author{
Jesús Lescano G. ${ }^{1,3}$, Ysaac Chipayo G. ${ }^{2}$, Miryam Quevedo U. ${ }^{1}$
}

\section{Resumen}

Se reporta el caso de una tortuga charapa Podocnemis expansa de 4 años de edad, que fue llevada a la consulta por presentar anorexia, vómitos y estreñimiento durante cuatro días. El animal era mantenido en un acuaterrario temperado y con sustrato de grava, con dieta a base de alimento comercial peletizado para tortugas acuáticas. No se observaron alteraciones al examen físico. Al examen radiológico se observaron múltiples cuerpos extraños radiopacos a nivel de estómago, diagnosticándose obstrucción gástrica por cuerpos extraños. Se administró tratamiento médico durante siete días con éxito limitado. Se realizó una gastrotomía, retirándose múltiples piedras de aproximadamente 5 x $10 \mathrm{~mm}$. Posteriormente, el animal continuó recibiendo tratamiento médico con drogas procinéticas, analgésicos y antibioticoterapia, recuperándose totalmente.

Palabras clave: tortuga charapa, quelonio, gastrotomía, drogas procinéticas, pica

\section{Abstract}

The case of a 4-year-old giant South American turtle (Podocnemis expansa) that presented anorexia, vomits and constipation during four days is reported. The animal was kept in a temperate land-water terrarium with gravel as substratum. The diet was only composed by commercial pelleted food for turtles. No alteration was observed during physical examination. Radiologic examination revealed multiple radio-opaque foreign

\footnotetext{
${ }^{1}$ Laboratorio de Anatomía Animal y Fauna Silvestre, ${ }^{2}$ Clínica de Animales Menores, Facultad de Medicina Veterinaria, Universidad Nacional Mayor de San Marcos, Lima, Perú

${ }^{3}$ E-mail: xtianlescano@gmail.com
}

Recibido: 20 de agosto de 2014

Aceptado para publicación: 23 de noviembre de 2014 
bodies in the stomach. Gastric foreign body obstruction was diagnosed. Medical therapy was administered during seven days with limited success. Gastrotomy was performed and multiple stones of about $5 \times 10 \mathrm{~mm}$ were removed. Then, the animal continued receiving medical therapy by means of prokinetic, analgesic and antibiotic drugs. The animal fully recovered.

Key words: giant South American turtle, chelonian, gastrotomy, prokinetic drugs, pica

\section{INTRODUCCIÓN}

El orden Testudines está conformado por más de 270 especies, que incluyen a las tortugas marinas, de río y terrestres (Rivera, 2003; McArthur et al., 2004; Norton, 2005; Schumacher, 2007). Una característica común para estos animales es la fuerte dependencia respecto a la temperatura ambiental, la cual es definida por diversos autores como poiquilotermia (temperatura corporal interna variante e igual a la temperatura ambiental), heliotermia (búsqueda de luz solar como fuente de calor corporal interno) y ectotermia (control de la temperatura interna mediante factores externos) (Boyer y Boyer, 1996; McArthur et al., 2004; Kik y Mitchell, 2005; Norton, 2005). Esta característica, sumada al hecho que ocupan una amplia variedad de ecosistemas, hace que las diversas especies de tortugas tengan requerimientos ambientales diferentes para mantener su metabolismo y salud (McArthur et al., 2004; Norton, 2005; Judah y Nuttall, 2008).

La tortuga charapa o arraú (Podocnemis expansa), también denominada tortuga gigante sudamericana, está clasificada en la categoría Preocupación Menor (LC) en la Lista Roja de Especies Amenazadas elaborada por la Unión Internacional para la Conservación de la Naturaleza (IUCN, por sus siglas en inglés) y en el Apéndice II de la Convención Internacional para el Comercio de Especies Amenazadas de Fauna y Flora Silvestres (CITES, por sus siglas en inglés) (Tortoise \& Freshwater Turtle Specialist
Group, 1996). Asimismo, es considerada como especie en Peligro de Extinción (EN) por el Gobierno Peruano (Ministerio de Agricultura, 2004; Ferronato y Morales, 2012).

Esta especie pertenece al suborden Pleurodira y es la tortuga dulceacuícola más grande de América del Sur (García, 2005; Rueda-Almonacid et al., 2007). Habita la mayoría de los ríos de las cuencas del Amazonas y el Orinoco, siendo registrada en Perú, Ecuador, Guyana, Trinidad y Tobago, Brasil, Bolivia, Venezuela y Colombia (Tortoise \& Freshwater Turtle Specialist Group 1996; Figueroa 2010).

La causa más frecuente de predisposición a enfermedad en tortugas mascota es nutrición deficiente o manutención inadecuada (McArthur et al., 2004; Longley, 2008). La constipación es uno de los trastornos gastrointestinales más frecuentes en tortugas cautivas (McArthur, 2004a; Oliveira et al., 2009) y usualmente es ocasionada por el consumo de material indigestible del sustrato (e.g. grava, arena, mazorca de maíz, cáscara de nueces triturada) (Boyer, 1996; McArthur, 2004c; Oliveira et al., 2009). Incidentalmente se pueden observar pequeñas cantidades de material extraño durante exámenes radiográficos sin signos clínicos de obstrucción gastrointestinal (Boyer, 1996; McArthur, 2004c; Büker et al., 2010). Asimismo, se reportan cuadros de obstrucción en ausencia de cuerpos extraños, posiblemente debido a presencia de neoplasias, intususcepción o vólvulo (McArthur, 2004c). 


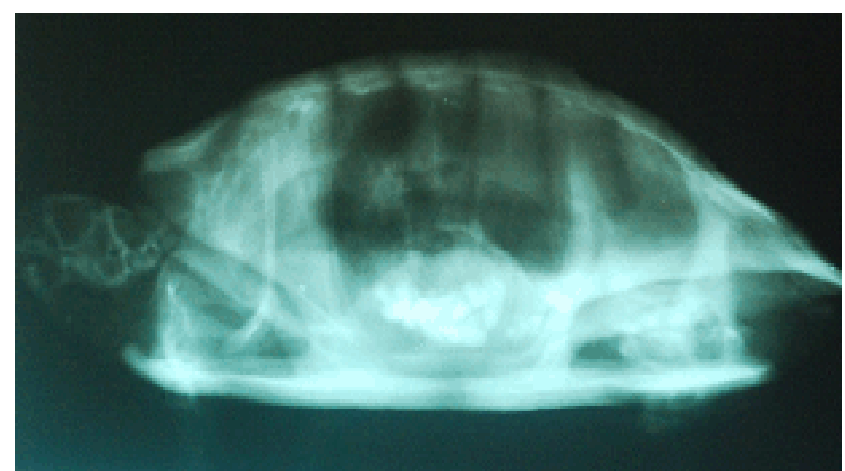

(a)

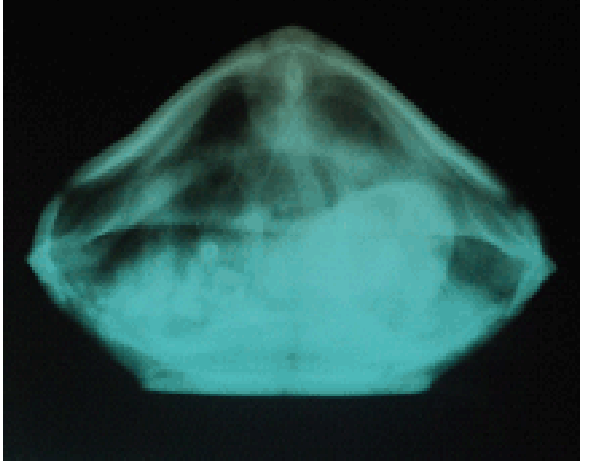

(b)

Figura 1. Radiografía de una tortuga Podocnemis expansa presentando numerosos cuerpos radiopacos a nivel del estómago e intestinos. (a) vista latero-lateral y (b) vista cráneocaudal

\section{Caso Clínico}

Una tortuga charapa (Podocnemis expansa), de aproximadamente 4 años de edad, se presenta a la consulta en la Clínica de Animales Silvestres y Exóticos de la Facultad de Medicina Veterinaria de la Universidad Nacional Mayor de San Marcos (Lima, Perú). El animal era mantenido en un acuaterrario de $60 \times 40 \times 40 \mathrm{~cm}$, temperado a $30{ }^{\circ} \mathrm{C}$ en su punto más caliente en tierra, con filtro y calefactor acuático y sustrato de grava. La dieta del animal consistía únicamente en alimento peletizado flotante para tortugas acuáticas.

Cuatro días antes de la consulta, el animal disminuyó considerablemente su apetito, y luego dejó de comer y defecar. Asimismo, el propietario observó que el animal había vomitado una sustancia blanquecina. Al examen físico, presentaba una condición corporal 3 de 5, $435 \mathrm{~g}$ de peso corporal, mucosas rosáceas y sin alteraciones visibles. En el examen radiológico de la cavidad celómica, se observó la presencia de numerosos cuerpos extraños radiopacos a nivel del estómago e intestino (Fig. 1), por lo que se diagnosticó obstrucción gastrointestinal.
Debido a la gran cantidad de material extraño observado en el tracto gastrointestinal, se sugirió realizar el tratamiento quirúrgico; sin embargo, el propietario solicitó realizar tratamiento médico como primera instancia. Se removió el sustrato de grava del acuaterrario y solo se colocaron rocas mayores de $10 \times 10 \times 10 \mathrm{~cm}$ como sustrato para las áreas secas. Se administró fluidoterapia de soporte empleando solución de Jarchow (también denominada "solución ringer reptil») por vía intracelómica, a dosis de $20 \mathrm{ml} / \mathrm{kg}$ c/24 h (Klingenberg, 1996; Norton, 2005). Además, se administró cisaprida ( $2 \mathrm{mg} / \mathrm{kg}$ PO $\mathrm{c} / 24 \mathrm{~h})$, metoclopramida (5 mg/kg PO c/24 h) y lactulosa $(0.5 \mathrm{ml} / \mathrm{kg} \mathrm{PO} \mathrm{c/8} \mathrm{h)} \mathrm{(Stein,} \mathrm{1996;}$ Franch y Martorell, 2001; Carpenter, 2005; Norton, 2005). Esta medicación fue administrada durante siete días.

Al tercer día de tratamiento, el propietario manifestó que la tortuga había defecado piedras de $5 \times 5 \mathrm{~mm}$. No se reportó la evacuación de más material extraño, de allí que al séptimo día de tratamiento, se procedió a instaurar el tratamiento quirúrgico.

Se administró acepromacina $(0.1 \mathrm{mg} / \mathrm{kg}$ $\mathrm{SC})$, tramadol (10 mg $/ \mathrm{kg} \mathrm{PO}$ ) y enrofloxacina (5 mg/kg SC) (Carpenter, 2005, Mosley, 2011; 

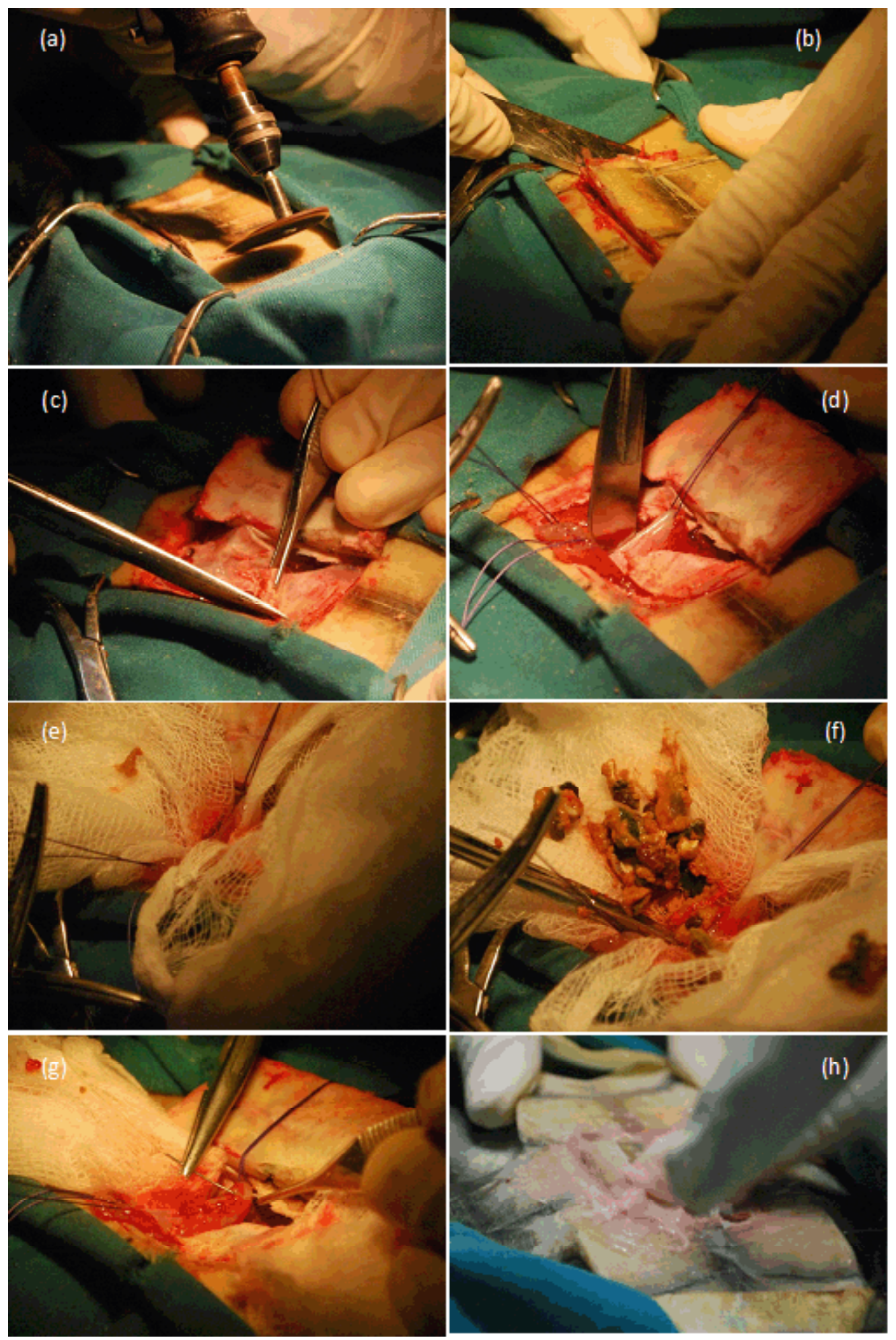

Figura 2. Cirugía en tortuga charapa. (a) Osteotomía del plastrón empleando una sierra rotativa y (b) martillo y cincel ortopédico; (c) Incisión de membrana celómica; (d) Colocación de puntos directrices en el estómago; (e) Colocación de paños de tercer campo; (f) Extracción de material extraño contenido en el estómago; (g) Sutura del estómago; (h) Aplicación de resina autopolimerizable sobre el plastrón 
Souza y Cox, 2011; Alves-Júnior et al., 2012; Sladky y Mans, 2012a; Sladky, 2014) como agentes pre-anestésicos. La inducción anestésica se hizo con ketamina $(80 \mathrm{mg} / \mathrm{kg}$ IM), midazolam ( $1 \mathrm{mg} / \mathrm{kg} \mathrm{IM})$ y xilacina $(0.5 \mathrm{mg} / \mathrm{kg}$ IM) (Carpenter, 2005). El mantenimiento anestésico fue realizado administrando isofluorano $2 \%$ vía endotraqueal (Carpenter, 2005; Sladky y Mans, 2012b). Asimismo, el paciente recibió soporte térmico y ventilación asistida durante todo el periodo anestésico y de recuperación (McArthur, 2004a; Sladky y Mans, 2012b; Schumacher y Mans, 2014).

En la antisepsia del plastrón se empleó solución 1:30 de clorhexidina $2 \%$ y embrocado con solución de yodopovidona 10\% (Bennett y Mader, 1996; McArthur y HernándezDivers, 2004). El abordaje de la cavidad celómica fue realizado mediante osteotomía trilateral del plastrón incidiendo sobre los escudos pectorales y abdominales, con una sierra rotativa, martillo y cincel ortopédicos (Fig. 2a,b) (Bennett y Mader, 1996; McArthur y Hernández-Divers, 2004; Oliveira et al., 2009). Posteriormente, se incidió la membrana celómica respetando la vasculatura (Fig. 2c) y se procedió a exteriorizar el estómago (McArthur y Hernández-Divers, 2004).

El estómago fue fijado empleando puntos directrices (Fig. 2d) y aislado del resto de órganos mediante paños de tercer campo (Fig. 2e) (Bennett y Mader, 1996). Se realizó una incisión en la curvatura mayor del estómago y se procedió a retirar el material extraño contenido (Fig. 2f). El estómago (Fig. $2 \mathrm{~g}$ ) y la membrana celómica fueron suturados empleando nylon con patrón de discontinuo simple (Rahal et al., 1998; Oliveira et al., 2009). El plastrón fue aproximado empleando resina acrílica autopolimerizable de metilmetracrilato (Fig. 2h). La herida fue protegida con gasa y vendaje elástico autoadherente.

El paciente olvió a ventilar por sí mismo, aproximadamente a los 45 minutos después de detener el flujo de isofluorano, y se recuperó completamente del evento anestésico aproximadamente 60 minutos más tarde.

El paciente recibió tratamiento médico posquirúrgico conformado por tramadol (10 $\mathrm{mg} / \mathrm{kg} \mathrm{PO} \mathrm{c} / 48 \mathrm{~h} \times 21$ días), flunixin meglumine $(1 \mathrm{mg} / \mathrm{kg} \mathrm{SC} \mathrm{c} / 24 \mathrm{~h} \mathrm{x} 3$ días), enrofloxacina ( $5 \mathrm{mg} / \mathrm{kg}$ PO $\times 7$ días), cisaprida ( $2 \mathrm{mg} / \mathrm{kg}$ PO c/24 h x 7 días), metoclopramida ( $5 \mathrm{mg} / \mathrm{kg}$ PO c/24 h x 7 días) y lactulosa (0.5 $\mathrm{ml} / \mathrm{kg}$ PO c/12 h x 5 días) (Stein, 1996; Franch y Martorell, 2001; Carpenter, 2005; Norton, 2005; Mosley, 2011; Souza y Cox, 2011; Sladky y Mans, 2012a; Sladky, 2014; Carpenter et al., 2014). De acuerdo al propietario, la tortuga volvió a comer normalmente al segundo día posterior a la cirugía. Asimismo, la tortuga evacuó algunas piedras con las heces.

\section{Discusión}

De acuerdo al conocimiento de los autores, es el primer caso en el país en que se reporta una obstrucción gastrointestinal por cuerpos extraños en una tortuga $P$. expansa mantenida en cautiverio. Al respecto, se han reportado algunos casos similares en tortugas pintadas Trachemys dorbignyi (Oliveira et al., 2009) y tortugas de orejas rojas Trachemys scripta elegans (Rahal et al., 1998).

Los signos clínicos asociados a obstrucción gastrointestinal en reptiles son bastante inespecíficos y pueden incluir anorexia, depresión, regurgitación, caquexia, debilidad, deshidratación (McArthur, 2004c). El paciente fue llevado a la consulta por presentar emesis, anorexia y constipación. Al respecto, la emesis puede deberse a parasitosis, septicemia, reacciones adversas a drogas, gastroenteritis y presencia de cuerpos extraños (Boyer, 1996; McArthur, 2004b). Por su parte, la anorexia, signo inespecífico de enfermedad en reptiles, puede estar asociada a enfermedad gastrointestinal, respiratoria, nutricional, metabólica, reproductiva y renal 
(Boyer, 1996; McArthur, 2004b); en tanto que la constipación puede deberse a diversos procesos patológicos. Considerando los signos clínicos inespecíficos que presentó el paciente, el uso de la radiografía como examen complementario fue de importancia crucial para lograr el diagnóstico (McArthur, 2004c; Büker et al., 2010).

La razón de la ingestión de piedras del sustrato del acuaterrario por el paciente no es clara, aunque el ambiente de tamaño reducido o carente de enriquecimiento ambiental puede contribuir al desarrollo de conductas anormales como pica (Rahal et al., 1998; Warwick et al., 2013). Según la descripción del propietario, el ambiente cumplía los requerimientos de temperatura y humedad, aunque probablemente no contaba con espacio suficiente para su tamaño. En este ambiente había estado por cuatro años y fue un ambiente diseñado para su tamaño inicial.

En el presente caso, los abordajes quirúrgico y médico de la obstrucción gastrointestinal fueron mutuamente complementarios. La limitada eficacia del tratamiento médico posiblemente se debió a la gran cantidad de material extraño obstructivo (McArthur, 2004c). La administración de fármacos procinéticos, después del tratamiento quirúrgico, conllevó a la evacuación de los restos de cuerpos extraños que no fueron extraídos durante la cirugía. Aquellos que no fueron removidos durante la ejecución de la gastrotomía, posiblemente fue debido a su ubicación en segmentos caudales del tracto digestivo que no fueron abordados quirúrgicamente.

\section{Literatura Citada}

1. Alves-Júnior JR, Bosso AC, Andrade MB, Jayme VdS, Werther K, Santos $A L$. 2012. Association of acepromazine with propofol in Giant Amazon Turtles Podocnemis expansa reared in captivity. Acta Cir Bras 27: 552-556.
2. Bennett RA, Mader DR. 1996. Soft tissue surgery. En: Mader DR (ed). Reptile medicine and surgery. Toronto: WB Saunders. p 287-298.

3. Boyer TH. 1996. Turtles, tortoises and terrapins (differential diagnosis by symptoms). En: Mader DR (ed). Reptile medicine and surgery. Toronto: WB Saunders. p 332-336.

4. Boyer TH, Boyer DM. 1996. Turtles, tortoises and terrapins (Biology). En: Mader DR (ed). Reptile medicine and surgery. Toronto: WB Saunders. p 6177.

5. Büker M, Foldenauer U, SimovaCurd S, Martig S, Hatt JM. 2010. Gastrointestinal obstruction caused by a radiolucent foreign body in a green iguana (Iguana iguana). Can Vet J 51: 511514.

6. Carpenter JW. 2005. Exotic animal formulary. $3^{\mathrm{a}}$ ed. USA: Elsevier Saunders. 564 p.

7. Carpenter JW, Klaphake E, Gibbons PM. 2014. Reptile formulary and laboratory normals. En: Mader DR, Divers SJ (eds). Current therapy in reptile medicine and surgery. San Luis, USA: Elsevier Saunders. p 382-410.

8. Ferronato BO, Morales VM. 2012. Biology and conservation of the freshwater turtles and tortoises of Peru. IRCF Reptiles \& Amphibians 19: 103-106.

9. Figueroa IC. 2010. Saber local, uso y manejo de las tortugas charapa Podocnemis expansa y taricaya Podocnemis unifilis (Testudines: Podocnemididae) en el resguardo Curare-Los Ingleses. La Pedrera: Amazonas: Colombia. Tesis de Maestría. Leticia, Colombia: Univ Nacional de Colombia. $287 \mathrm{p}$.

10. Franch J, Martorell J. 2001. Intestinal obstruction in a savannah monitor. Contin Educ Pract Vet 23(12): 10831087.

11. García N. 2005. Biología reproductiva y conservación de las tortugas charapa Podocnemis expansa, cupiso Podocnemis sextuberculata y taricaya Podocnemis unifilis en las playas ale- 
dañas al Municipio de Puerto Nariño (Amazonas). Tesis de Ecología. Colombia: Facultad de Estudios Ambientales y Rurales, Pontificia Universidad Javeriana. 209 p.

12. Judah V, Nuttall K. 2008. Reptiles. En: Judah V, Nuttall K (eds). Exotic animal care and management. Canadá: Thomson Delmar Learning. p 155-190.

13. Kik MJL, Mitchel MA. 2005. Reptile cardiology: a review of anatomy and physiology, diagnostic approaches, and clinical disease. Semin Avian Exot Pet 14: 52-60.

14. Klingenberg RJ. 1996. Therapeutics. En: Mader DR (ed). Reptile medicine and surgery. Toronto: WB Saunders. p 299321.

15. Longley L. 2008. Chelonian (tortoise, terrapin and turtle) anaesthesia. En: Longley L (ed). Anaesthesia of exotic pets. China: Saunders Elsevier. p 228237.

16. McArthur S. 2004a. Anaesthesia, analgesia and euthanasia. En: McArthur S, Wilkinson R, Meyer J (eds). Medicine and surgery of tortoises and turtles. Oxford: Blackwell Publishing. p 379-401.

17. McArthur S. 2004b. Interpretation of presenting signs. En: McArthur S, Wilkinson R, Meyer J (eds). Medicine and surgery of tortoises and turtles. Oxford: Blackwell Publishing. p 273-300.

18. McArthur S. 2004c. Problem-solving approach to common diseases of terrestrial and semi-aquatic chelonians. En: McArthur S, Wilkinson R, Meyer J (eds). Medicine and surgery of tortoises and turtles. Oxford: Blackwell Publishing. p 309-377.

19. McArthur S, Hernández-Divers $S$. 2004. Surgery. En: En: McArthur S, Wilkinson R, Meyer J (eds). Medicine and surgery of tortoises and turtles. Oxford: Blackwell Publishing. p 403-464.

20. McArthur S, Wilkinson R, Barrows $M$, Meyer J. 2004. Introduction. En: McArthur S, Wilkinson R, Meyer J (eds). Medicine and surgery of tortoises and turtles. Oxford: Blackwell Publishing. p 1-30.

21. Ministerio de Agricultura. 2004. Decreto Supremo N ${ }^{\circ}$ 034-2004-AG. El Peruano: 276853-276855.

22. Mosley C. 2011. Pain and nociception in reptiles. Vet Clin North Am Exot Anim Pract 14: 45-60. doi: 10.1016/ j.cvex.2010.09.009

23. Norton TM. 2005. Chelonian emergency and critical care. Semin Avian Exot Pet 14: 106-130. doi: 10.1053/ j.saep.2005.04.005

24. Oliveira FS, Delfini A, Martins LL, Farla Jr.D, Machado MRF. 2009. Obstrução intestinal e enterotomia em tigre d'água (Trachemys dorbignyi). Acta Sci Vet 37: 307-310.

25. Rahal SC, Teixeira CR, Castro GB, Vulcano LC. 1998. Intestinal obstruction by stones in a turtle. Can Vet J 39: 375-376.

26. Rivera S. 2003. The chelonians. En: Ballard B, Cheeck R (eds). Exotic animal medicine for the veterinary technician. Ames: Iowa State Press. p 129-144.

27. Rueda-Almonacid JV, Carr JL, Mittermeier RA, Rodríguez-Mahecha $J V$, Mast RB, Vogt RC, et al. 2007. Las tortugas y los cocodrilianos de los países andinos del trópico. Serie de guías tropicales de campo N. ${ }^{\circ}$ 6. Conservación Internacional. Bogotá: Ed Panamericana. $538 \mathrm{p}$.

28. Schumacher J. 2007. Chelonians (turtles, tortoises and terrapins). En: West G, Heard D, Caulkett N (eds). Zoo animal and wildlife immobilization and anesthesia. Iowa, UA: Blackwell Publishing. p 257-266.

29. Schumacher J, Mans C. 2014. Anesthesia. En: Mader DR, Divers SJ (eds). Current therapy in reptile medicine and surgery. San Luis, USA: Elsevier Saunders. p 134-153.

30. Sladky KK. 2014. Analgesia. En: Mader DR, Divers SJ (eds). Current therapy in reptile medicine and surgery. San Luis, USA: Elsevier Saunders. p 217-228. 
31. Sladky KK, Mans C. 2012a. Clinical analgesia in reptiles. J Exot Pet Med 21: 158-167. doi: 10.1053/j.jepm. 2012. 02.012

32. Sladky KK, Mans C. 2012b. Clinical anesthesia in reptiles. J Exot Pet Med 21: 17-31. doi: 10.1053/j.jepm. 2011. 11.013

33. Stein $G$ 1996. Reptile and amphibian formulary. En: Mader DR (ed). Reptile medicine and surgery. Toronto: WB Saunders. p 465-472.

34. Souza MJ, Cox SK. 2011. Tramadol use in zoologic medicine. Vet Clin North Am
ExotAnim Pract 14: 117-130. doi: 10.1016/ j.cvex.2010.09.005

35. Tortoise \& Freshwater Turtle Specialist Group. 1996. Podocnemis expansa. En: IUCN 2013. IUCN Red List of Threatened Species. Version 2014.3. [Internet]. Disponible en: http:// www.iucnredlist.org/details/17822/0

36. Warwick C, Arena P, Lindley S, Jessop M, Steedman C. 2013. Assessing reptile welfare using behavioural criteria. In Practice 35: 123-131. 